\title{
HPV 16 E6 (18-26) Peptide
}

National Cancer Institute

\section{Source}

National Cancer Institute. HPV 16 E6 (18-26) Peptide. NCI Thesaurus. Code C38117.

A peptide derived from the oncogenic human papillomavirus (HPV) early gene product E6. The HPV 16 transforming protein E6 is expressed in precancerous and malignant cervical lesions and has a high affinity for the most common human lymphocyte antigen (HLA), HLA-A2. Immunogenic peptides from the HPV 16 E6 may be used to trigger a Tcell-mediated immune response to HPV. (NCI04) 